\title{
Design Thinking Makes Great Ideas Viable
}

\section{Brian Janz (University of Memphis)}

KEYWORDS: Entrepreneurship, Innovation, Teaching Methods, student entrepreneurs, Innovators, Student innovators.

Entrepreneurs often start their business with a "good" idea they have that has not been properly vetted by potential customers. As a result, entrepreneurs often invest a great deal of time, effort, and money in building out their idea, only to find that it falls short when facing the "acid test" of the market.

Design Thinking (often referred to as "Human-Centered Design") is a design/development process that helps you understand your potential customers' real wants and needs - at a deep, often emotional level. It includes an empathy-based learning process followed by radical ideation, rapid prototyping and effective storytelling. The process basics can be taught quickly utilizing the Stanford Design School's (the "D School's") "gift giving" exercise. All materials and instructions for teaching can be found at their website:

https://dschool.stanford.edu/resources/gear-up-how-tokick-off-a-crash-course.

In facilitating this exercise dozens of times over the years, I have learned the following things:

1. The exercise typically takes 90 minutes to complete, including a few minutes at the end to debrief what was learned;

2. The exercise can be used in the classroom, in the community, or wherever two or more interested people (plus a facilitator) can be found;

3. The exercise is designed to be completed by pairs of participants. Odd numbers can work, just not as cleanly;

4. The exercise makes sense for most age groups, from teenagers on up. Facilitators with experience working with children could easily figure out how to slightly adapt the process to work with grade-school children as well;

5. Gift-giving is a universal experience that resonates with all people regardless of age, race, country of origin, or culture, so it represents a relevant and relatable challenge;

6. Background music helps facilitate the activities, especially when the participants are working with each other;

7. The exercise can be completed with just paper and pencil, however, it is best done with at least some basic craft supplies. I have found that tape, pipe cleaners, construction paper (and scissors), and maybe some popsicle sticks are the most important. Anything more than this is a bonus.

I have conducted the exercise as part of a graduate MBA IT course (in one class session), as it helps to introduce the concepts of systems development -including information requirements gathering, working with customers/end-users, brainstorming and prototyping. I have also conducted it as part of an undergraduate entrepreneurial/innovation course to teach design thinking, and to encourage creativity and innovative thought. Here, I use the exercise to kick off the course and introduce Design Thinking and then use the rest of the semester to more deeply cover the concepts and work on a semester-long design project. I have conducted it in several corporate and community settings to better prepare participants to understand the challenges they face in the community or organization, and to better help them develop their ideas to address the challenges. In all cases, the exercise is fine by itself, or it serves as a primer for follow-on detailed discussions on innovation, ideation, prototyping, business model/canvas development or project kick-off. It is well-received wherever it is conducted.

Additional Search Terms: entrepreneurship courses, teaching ideas, teaching resources, classroom ideas, entrepreneurship classes, business schools, business school classes, entrepreneurship students, professors 